\title{
Aggressive ameloblastic fibro-odontoma assessment with CBCT and treatment
}

\author{
P. S. C. Lúcio $\cdot$ R. B. Cavalcante $\cdot$ R. N. Maia • \\ E. S. Santos · G. P. Godoy
}

Received: 29 October 2012/ Accepted: 21 February 2013/Published online: 30 April 2013

(C) European Academy of Paediatric Dentistry 2013

\begin{abstract}
Background Ameloblastic fibro-odontomas (AFO) are rare lesions and defined by the World Health Organisation as a tumour composed of a proliferation of odontogenic epithelium immersed in ectomesenchyme reminiscent of the interdental papilla. It also presents inductive processes leading to formation of enamel and dentine, confusing, histopathologically, with odontoma. Despite numerous efforts, there is still considerable confusion over its controversial aetiopathogenesis and treatment. A brief review of the literature on the clinical, pathological and therapeutic features of this lesion is reported.

Case report A case of aggressive AFO in a 3-year-old boy was referred to the Oral Diagnostics Service of Hospital General de Fortaleza for evaluation following a 3-months painless swelling in the right mandibular and facial regions. During anamnesis, the patient's carers reported no systemic problem and his medical history was non-contributory. Intra-orally there was a swelling of the
\end{abstract}

P. S. C. Lúcio · G. P. Godoy

Department of Dentistry, University Estadual da

Paraíba, Campina Grande, Brazil

R. B. Cavalcante $\cdot$ E. S. Santos

Department of Dentistry, University of de Fortaleza

(UNIFOR), Fortaleza, Brazil

R. N. Maia

Department of Oromaxillofacial Surgery, General Hospital

of Fortaleza, Fortaleza, Brazil

G. P. Godoy $(\bowtie)$

Departamento de Odontologia Programa de Pós-Graduação em

Odontologia, Universidade Estadual da Paraíba, Av. das

Baraúnas, s/n Bodocongó, Campina Grande, PB, Brazil

e-mail: gruiga@hotmail.com right lateral portion of the mandible, adjacent teeth were not mobile and the oral mucosa appeared normal and showed no signs of ulceration. Radiographic examination, with panoramic radiography and cone beam computed tomography with three-dimensional (3D) reconstruction, was better able to identify the extent of bone involvement and showed an expansive hypodense image in the right mandibular region. 3D reconstruction showed expansion of buccal and lingual cortical bone and the preservation of basal bone.

Treatment Enucleation and curettage of the lesion were carried out and care was taken not to fracture the basal bone during the surgical procedure. Tissue specimens were sent for histopathologic analysis. Aspiration performed during surgery was negative for blood and other exudates. Follow-up No recurrence has occurred during a followup period of 11 months.

Conclusion A comprehensive diagnosis including all clinical, radiographic and histopathologic features is necessary for the success of therapy, which varies from case to case, thus, improving the quality of life of patients with AFO.

Keywords Ameloblastic fibro-odontoma - Mandible . Odontogenic tumours · Therapeutics · Cone beam computed tomography

\section{Background}

Ameloblastic fibro-odontoma (AFO) is defined by the World Health Organisation (WHO) as an odontogenic tumour composed of proliferating odontogenic epithelium immersed in ectomesenchyme that resembles the interdental papilla. This tumour is, therefore, similar to ameloblastic fibroma, but is associated with inductive 
changes that lead to the formation of enamel and dentine (Barnes et al. 2005; Nascimento et al. 2009; Riu et al. 2010). AFO is rare, accounting for $3.1 \%$ of all odontogenic tumours (Reis et al. 2007; Santos et al. 2011).

AFO commonly affects children at an average age of 8-12 years and has no preference for gender or anatomical site, although some studies have shown a higher frequency of the tumour in the posterior region of the mandible (Reis et al. 2007; Rebelo-Pontes et al. 2012). AFOs are generally asymptomatic slow-growing tumours, although an aggressive behaviour has been reported. Radiographically, AFO appears as a radiolucent, uni- or multilocular, well-delimited area with central radiopacity of variable size and shape, findings compatible with a dental structure that can be associated with an unerupted tooth (Reis et al. 2007; Nascimento et al. 2009).

Treatment consists of conservative removal of the entire lesion, which, because it is well delimited (presence of a fibrous capsule), can be easily distinguished from the surrounding bone tissue. The prognosis is excellent and recurrence and malignant transformation are rare findings (Riu et al. 2010; Santos et al. 2011; Rebelo-Pontes et al. 2012). The case described herein reports the unusual presentation of aggressive AFO in a 3 -year-old child. The clinical, radiographic, histopathologic, and therapeutic features of this tumour are discussed.

\section{Case report}

A 3-year-old boy was referred to the Oral Diagnostics Service of Hospital General de Fortaleza for evaluation of a 3-months painless swelling in the right mandibular and facial region. During anamnesis, the child's carers reported no systemic problem and his medical history was noncontributory.

\section{Extra-oral physical examination}

This revealed a swelling in the posterior region of the mandibular body, angle and ramus, which extended to the condylar neck and coronoid apophysis on the right side, causing severe facial asymmetry. The skin covering the area had a normal colour.

Intra-oral examination

This showed a swelling of the right lateral portion of the mandibule. The adjacent teeth were not mobile and the oral mucosa appeared normal and showed no signs of ulceration (Fig. 1).
Radiographic examination

Panoramic radiography and cone beam computed tomography $(\mathrm{CBCT})$ with three-dimensional (3D) reconstruction were performed to better identify the extent of bone involvement. Panoramic radiography showed a radiolucent, multilocular image measuring approximately $10 \mathrm{~cm}$ involving part of the mandibular body and the entire right mandibular ramus and angle. An unerupted tooth was observed inside the area, as well as small radiopaque foci (Fig. 2). CBCT revealed an expansive hypodense image in the right mandibular region, in addition to material of variable density that was compatible with dental structures and the presence of an unerupted tooth. 3D reconstruction demonstrated expansion of buccal and lingual cortical bone and the preservation of basal bone (Fig. 2).

\section{Treatment}

Treatment consisted of enucleation and curettage of the lesion, under general anaesthesia (GA) and care was taken not to fracture the basal bone during the surgical procedure. An intra-oral incision was made from the mandibular ridge of the angle to the area of the canine, the mucosa was separated, and the tumour was excised completely (excisional biopsy). The specimens were sent for histopathologic analysis. Aspiration performed during surgery was negative for blood and other exudates. Gross inspection showed a pale pink tumour measuring $10 \mathrm{~cm}$ along its major axis, a fibrous capsule containing calcifications, and an associated unerupted tooth. The diagnostic hypothesis was ameloblastic fibroma and AFO.

\section{Histological examination}

Microscopically, proliferation of islands and cords of anastomosed odontogenic epithelium were observed (Fig. 3a). The peripheral columnar epithelial cells presented hyperchromatic nuclei and a palisade arrangement. A loose arrangement resembling the stellate reticulum of the enamel organ was observed in the centre of the tumour (Fig. 3b). The mesenchyme of the lesion was highly cellularised, containing spherical, stellate and/or spindleshaped cells. Eosinophilic amorphous material compatible with dentinoid material was also observed (Fig. 3c, d). These histopathological findings led to the diagnosis of AFO.

\section{Follow-up}

The patient presented no complications during the postoperative period, with good surgical results. To date, no 

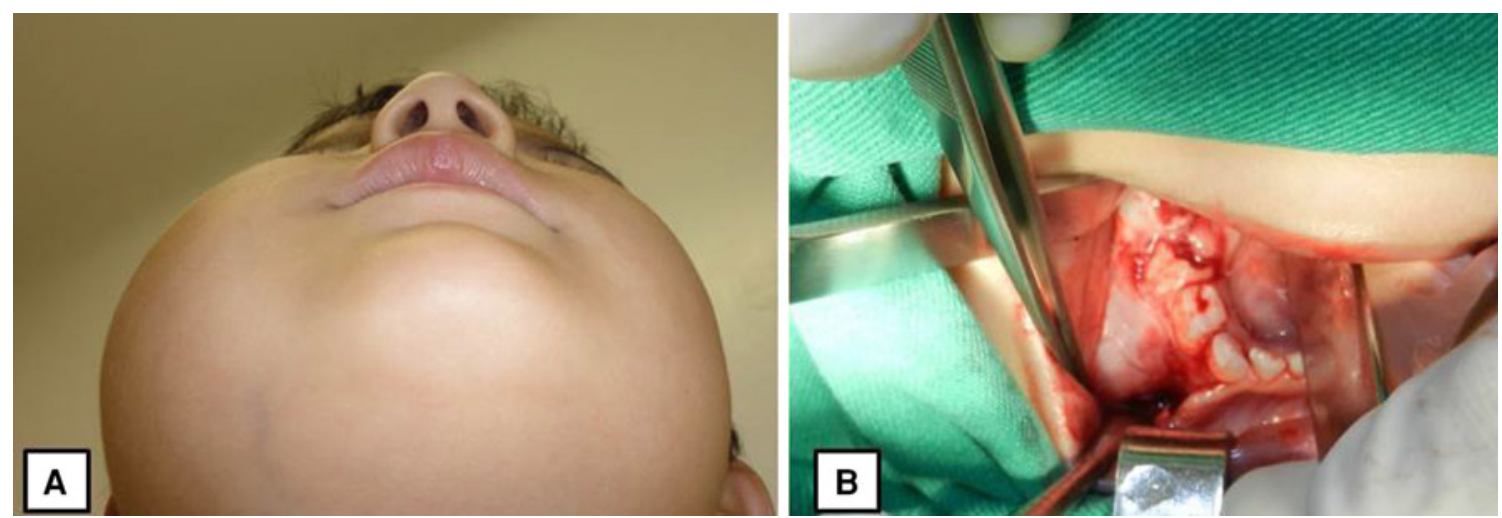

Fig. 1 Photographs illustrating a case of ameloblastic fibro-odontoma: a extra-oral physical examination; b intra-oral showing expansion of body of the mandible

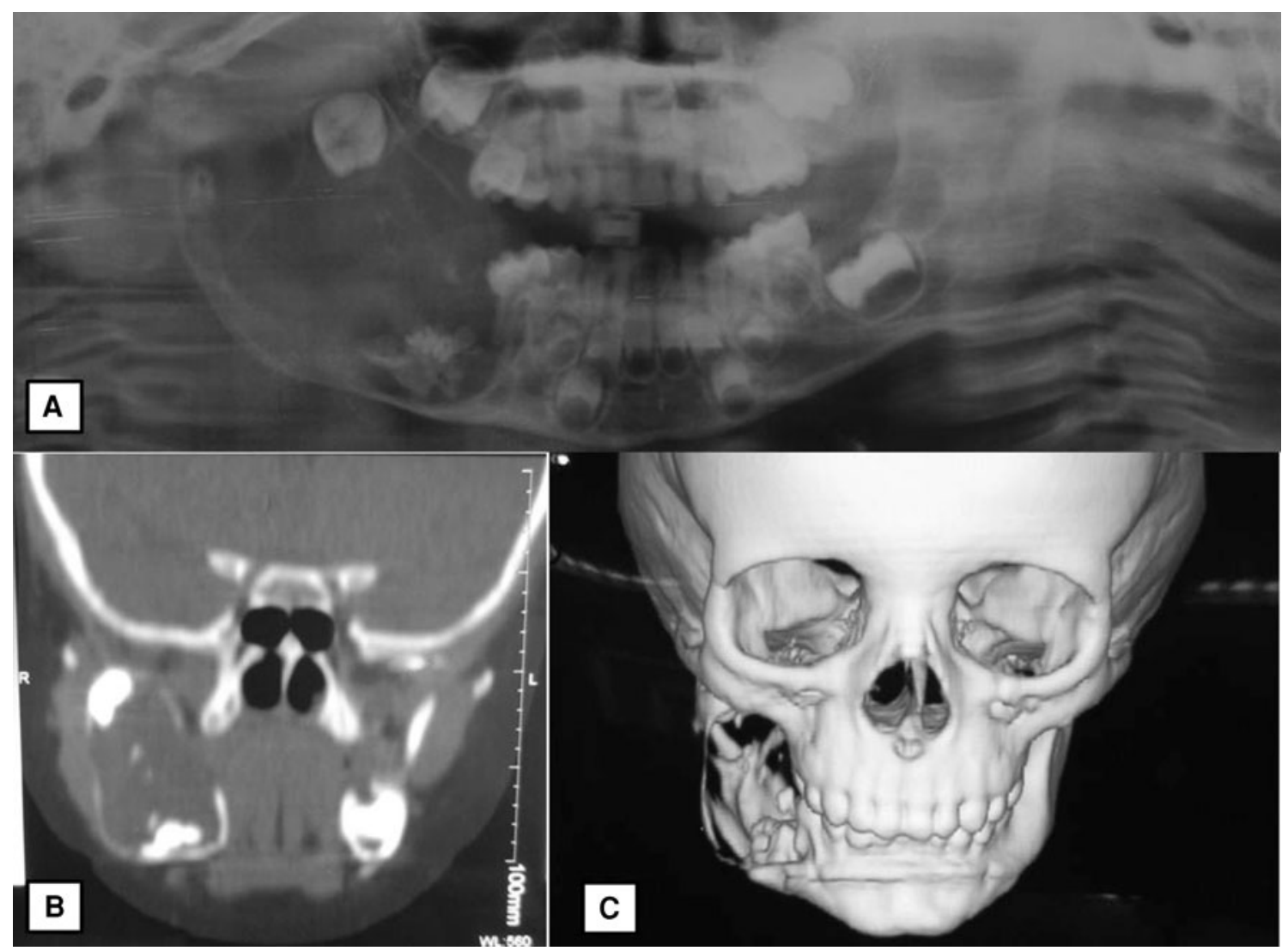

Fig. 2 Radiographic and imaging assessments of a case of ameloblastic fibro-odontoma showing: a panoramic radiograph, b imaging test and $\mathbf{c} 3 \mathrm{D}$ reconstruction revealing extensive osteolytic lesion in the posterior right mandible

recurrence has been observed during a follow-up period of 11 months.

\section{Discussion}

Ameloblastic fibro-odontoma is a relatively rare, benign odontogenic tumour whose aetiopathogenesis is controversial. Its correct classification continues to be a matter of discussion, particularly the distinction between hamartoma and neoplasm (Rebelo-Pontes et al. 2012). According to Riu et al. (2010), AFO is a combination of complex odontoma and ameloblastoma with a benign biological behaviour similar to that of ameloblastic fibroma. In contrast to the latter, the term "ameloblastic odontoma" refers to tumours characterised by the histopathologic 

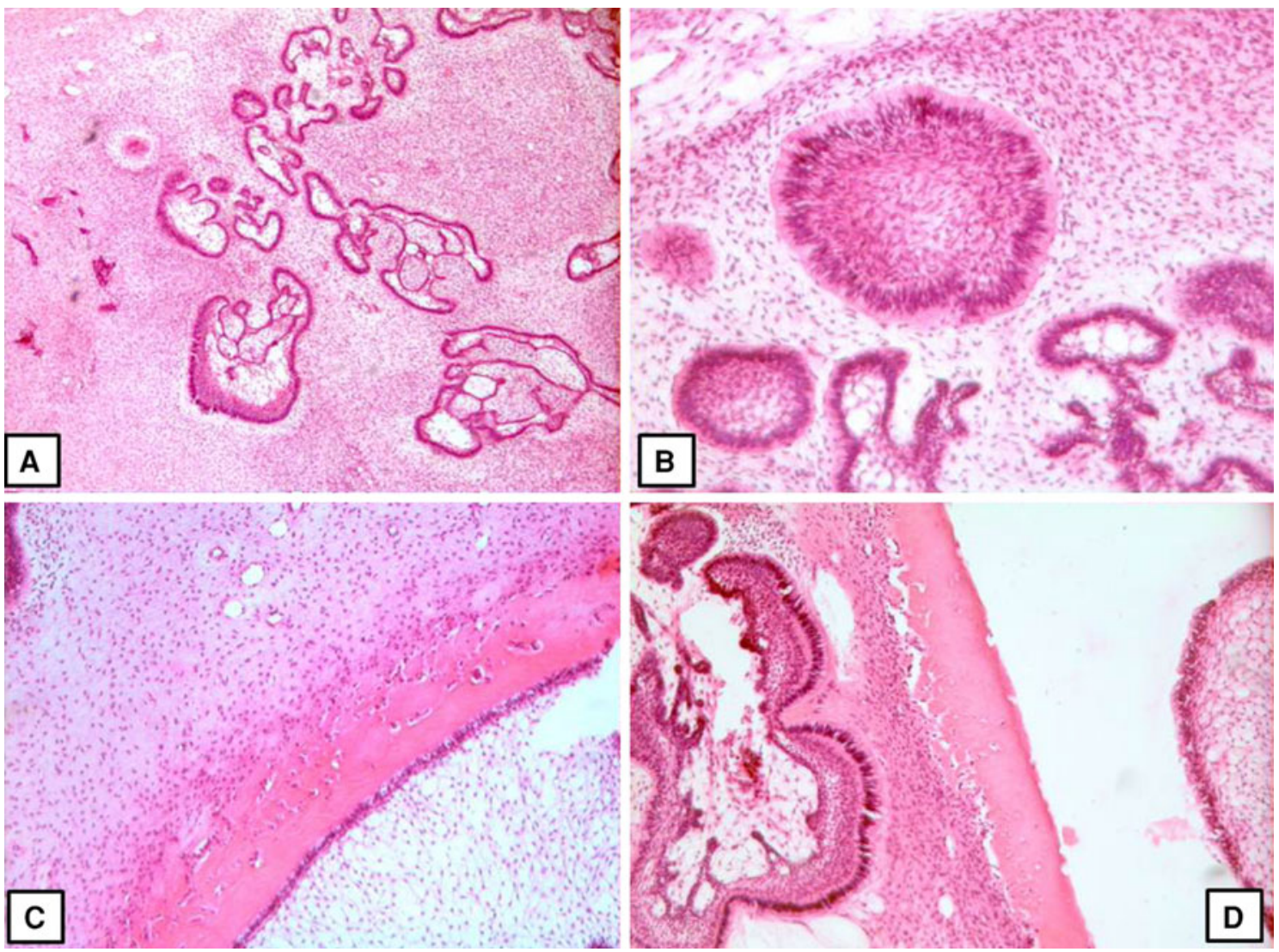

Fig. 3 Photomicrograph of ameloblastic fibro-odontoma lesion: a proliferation of islands, nests and cords of epithelial cells in the mesenchyme richly cellularised; b peripheral hyperchromatic columnar cells arranged in palisades and with loose central organisation,

similar to the stellate reticulum of the enamel organ; $\mathbf{c}$ and d amorphous eosinophilic tissue compatible with dentine-like formation (Haematoxylin and eosin stain, $\times 40$ )

combination of complex odontoma and ameloblastoma. These tumours may therefore be locally aggressive, destroying bone and soft tissue. Philipsen et al. (1997) and Slootweg (1981) suggested AFO to present hamartomatous features, whereas ameloblastic fibroma exhibits a neoplastic nature. Most authors agree that AFO is a separate entity. However, in some situations this tumour can be histopathologically indistinguishable from immature complex odontoma. Therefore, the relative arrangement of soft tissues and the developmental stage of the involved tooth can be useful criteria for the diagnosis (Correa-Pontes et al. 2008; Santos et al. 2011).

Studies suggest that ameloblastic fibroma, AFO and odontoma (compound and complex) are evolutionary stages of the same lesion (Soares et al. 2006; Neville et al. 2009; Tolentino et al. 2010), or that ameloblastic fibroma and AFO are precursor stages of odontoma (Rebelo-Pontes et al. 2012). However, the three lesions represent distinct entities. The ameloblastic fibroma and AFO exhibits a more locally aggressive behaviour and are associated the bone expansion. This feature is not observed in complex odontomas which therefore have a more indolent behaviour (Correa-Pontes et al. 2008).

The age of the present patient and location of the tumour were similar to those reported in the literature (Table 1). An interesting finding was the more aggressive behaviour of the tumour as demonstrated by extensive bone destruction, indicating a true neoplastic behaviour and thus being compatible with AFO.

The differential diagnosis between AFO and ameloblastic fibroma is made based on the presence or absence of enamel and dentine (Soares et al. 2006; Santos et al. 2011). However, other tumours exhibiting mixed imaging features should also be taken into account, such as calcifying epithelial odontogenic tumour, calcifying cystic odontogenic tumour, complex odontoma, and adenomatoid odontogenic tumour (Correa-Pontes et al. 2008; Zouhary et al. 2008).

In the present case, the ectomesenchymal portion was highly cellularised and the cells had distinct shapes (spherical, stellate, spindle-shaped), a fact indicating significant cellular activity. This finding may explain the more aggressive biological behaviour observed in the present study, in agreement with the cases reported by Reichart et al. (2004), Soares et al. (2006), and Reis et al. (2007).

Conservative surgery combined with curettage is the treatment of choice for AFO because of the benign 


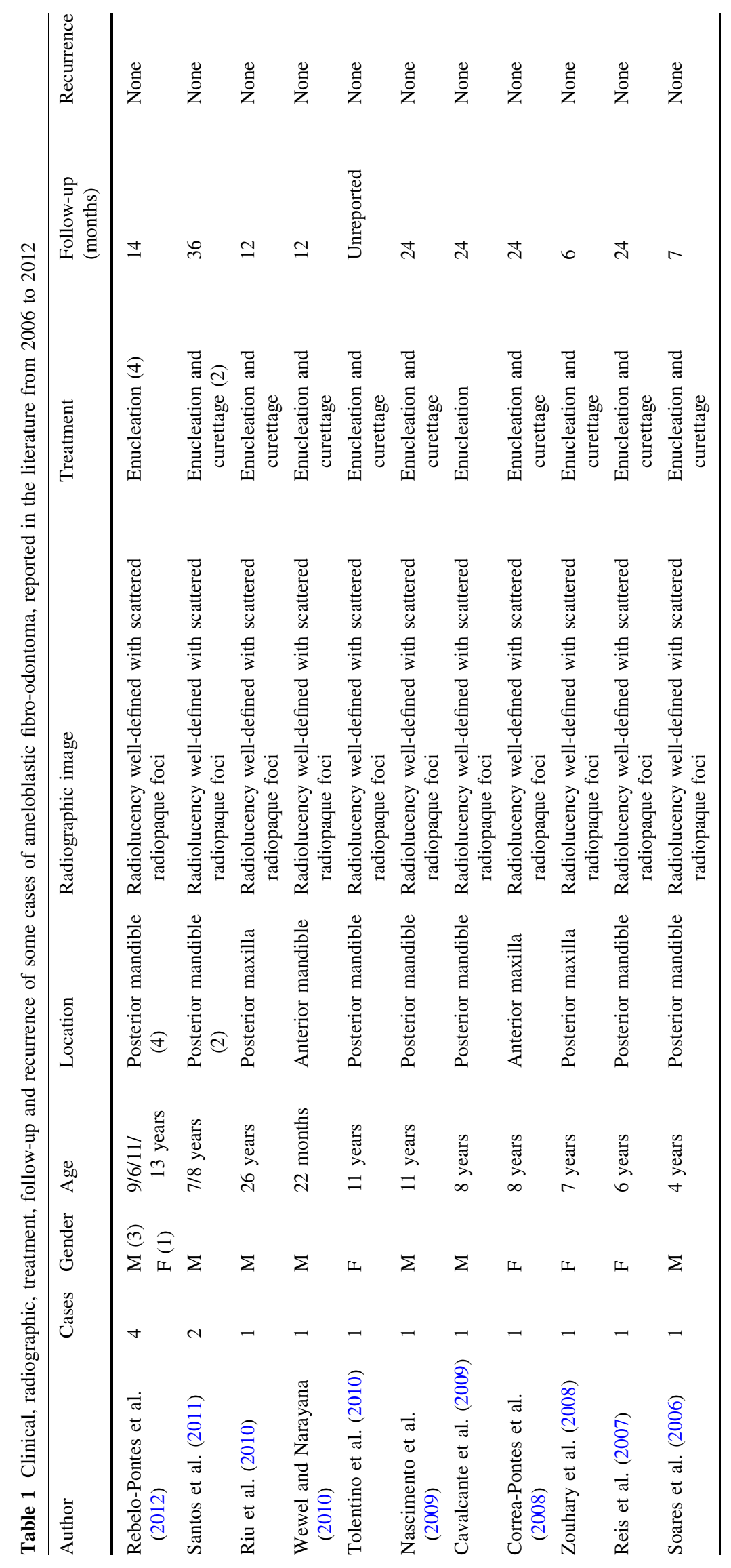


behaviour of the tumour. However, no consensus exists regarding whether or not to preserve associated teeth (Santos et al. 2011; Rebelo-Pontes et al. 2012). In some situations, surgical enucleation of AFO and preservation of any teeth involved are adopted. In these cases, spontaneous tooth eruption and the absence of local recurrence should be evaluated after 24 months of follow-up (Okamura et al. 1992). However, some authors argue that maintenance of any involved teeth may impair complete removal of the neoplastic tissue, with the persistence of tumour cells that can lead to recurrence (Miller et al. 1976; Friedrich et al. 2001; Chang et al. 2002).

Nevertheless, regardless of whether or not the tooth is maintained, recurrence of AFO is associated with inadequate surgical excision and occurs if tumour remnants persist at the resection margins or in the involved tooth, particularly in the case of large tumours. Changes in the histopathologic pattern of the tumour consisting of a more disorganised fibrous stroma and displacement of the epithelial component are observed in these cases (Friedrich et al. 2001). In the present study, complete tumour enucleation and removal of the involved teeth were chosen because of the extent of the tumour and bone involvement. No local recurrence was observed during a follow-up period of 11 months.

\section{Conclusion}

Although a considerable improvement has been observed since presentation as an unusual and aggressive case, a cure is not assured. However, in the absence of recurrence up to the present time, we are optimistic that this will continue to be the case for a long time. A comprehensive diagnosis including all clinical, radiographic and histopathological features was necessary for the successful treatment enhanced by the use of CBCT. Clinical management of such patients varies from case to case, thus improving the quality of life of patients with AFO. In addition, the present case supports the view that AFO is of neoplastic rather than hamartomatous nature.

\section{References}

Barnes L, Eveson JW, Reihcart P, Sidransky D. World Health Organization international histological classification of tumours. Lyon: IARC Press; 2005.
Cavalcante AS, Anbinder AL, Costa NC, Lima JR, Carvalho YR. Ameloblastic fibro-odontoma: a case report. Med Oral Patol Oral Cir Bucal. 2009;14(12):650-3.

Chang H, Precious DS, Shimizu MS. Ameloblastic fibro-odontoma: a case report. J Can Dent Assoc. 2002;68(4):243-6.

Correa-Pontes FS, Pontesa HA, Sá Nogueira JE. Ameloblastic fibroodontoma: case report with maintenance of the involved teeth. Int J Pediatr Otorhinolaryngol. 2008;3(1):48-52.

Friedrich RE, Siegert J, Donath K, Jakel KT. Recurrent ameloblastic fibro-odontoma in a 10-year-old boy. J Oral Maxillofac Surg. 2001;59(11):1362-6.

Miller AS, Lopez CF, Pullon PA, Elzay RP. Ameloblastic fibroodontoma. Report of seven cases. Oral Surg Oral Med Oral Pathol. 1976;41(3):354-65.

Nascimento JE, Araújo LJ, Almeida LY, De-Paula AM, Bonan PR. Ameloblastic fibro-odontoma: a conservative surgical approach. Med Oral Patol Oral Cir Bucal. 2009;14(12):654-7.

Neville BW, Damm DD, Allen CM, Bouquot JE. Oral and maxillofacial pathology. New York: Elsevier; 2009.

Okamura M, Nakahara H, Matsuya T. Treatment of ameloblastic fibro-odontoma without removal of the associated impacted permanent tooth: report of cases. J Oral Maxillofac Surg. 1992; 50(10):1094-7.

Philipsen HP, Reichart PA, Praetorius F. Mixed odontogênico tumour and odontomas. Considerations on interrelationship. Review of the literature and presentation of 134 new cases of odontomas. Oral Oncol. 1997;33(2):86-99.

Rebelo-Pontes HA, Correa-Pontes FS, Lameira AG, et al. Report of four cases of ameloblastic fibro-odontoma in mandible and discussion of the literature about the treatment. J Craniomaxillofac Surg. 2012;40(2):59-63.

Reichart P, Philipsen H, Gelderblom H, Stratmann U. Ameloblastic fibro-odontoma-report of two cases with ultrastructural study of tumour dental hard structures. Oral Oncol. 2004;40(1):8-12.

Reis SR, Freitas CE, Espírito-Santo AR. Management of ameloblastic fibro-odontoma in a 6-year-old girl preserving the associated impacted permanent tooth. J Oral Sci. 2007;49(4):331-5.

Riu GD, Meloni SM, Contini M, Tullio A. Ameloblastic fibroodontoma: case report and review of the literature. J Craniomaxillofac Surg. 2010;38(2):141-4.

Santos TS, Carvalho RW, Avelar RL, et al. Ameloblastic fibroodontoma in children: report of 2 cases. J Dent Child. 2011; 78(3):173-7.

Slootweg PJ. An analysis of the interrelationship of the mixed odontogenic tumours-ameloblastic fibroma, ameloblastic fibroodontoma, and the odontomas. Oral Surg Oral Med Oral Pathol. 1981;51(3):266-76.

Soares RC, Godoy GP, Neto JC, Batista L, Pinto LP. Ameloblastic fibro-odontoma: report of a case presenting an unusual clinical course. Int J Pediatr Otorhinolaryngol. 2006;1(3):200-3.

Tolentino ES, Centurion BS, Lima MC, et al. Ameloblastic fibro odontoma: a diagnostic challenge. Int J Dent. 2010;2010:1-5.

Wewel J, Narayana N. Ameloblastic fibro-odontoma of the anterior mandible in a 22-month-old boy. Indian J Dent Res. 2010; 21(4):618-20.

Zouhary KJ, Said-Al-Naief N, Waite PD. Ameloblastic fibro-odontoma: expansile mixed radiolucent lesion in the posterior maxilla: a case report. Oral Surg Oral Med Oral Pathol Oral Radiol Endod. 2008;106(4):15-21. 